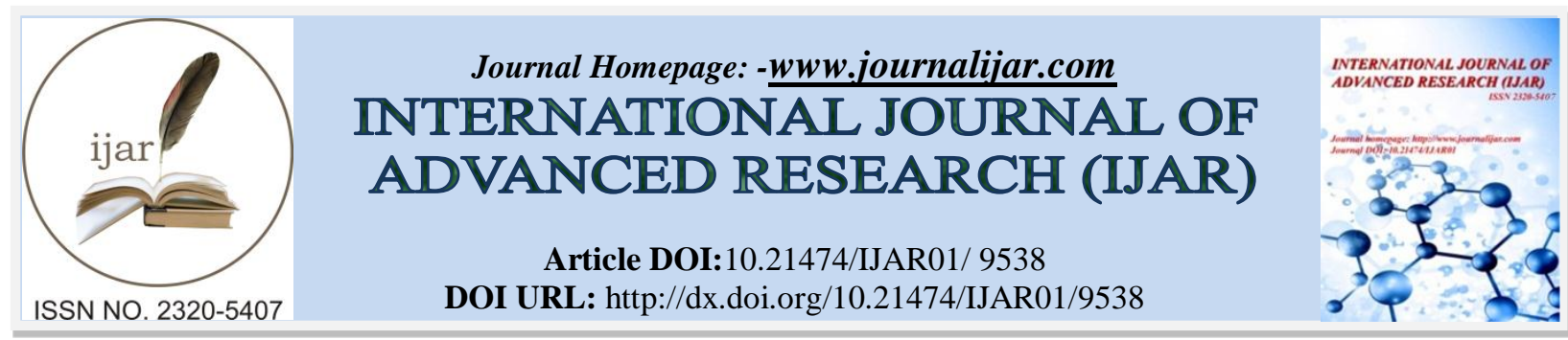

RESEARCH ARTICLE

\title{
PRISE EN CHARGE CHIRURGICALE D'UN CARCINOME EPIDERMOIDE DU TALON.SURGICAL MANAGEMENT OF A SQUAMOUS CELL CARCINOMA OF THE HEEL.
}

\section{J. Hafidi ${ }^{12}$, M.A. Mokfi ${ }^{1}$, M. Raboune ${ }^{1}$, S. El Mazouz ${ }^{1}$, N. Gharib ${ }^{1}$, A. Abbassi ${ }^{1}$, S. El Khloufi ${ }^{2}$, M.L Bouchikhi $^{2}$, M. Jiddane ${ }^{2}$ and Y. Bjijou' ${ }^{2}$.}

1. Service de chirurgie plastique et des brûlés, hôpital Ibn-Sina, Rabat.

2. Laboratoire d'anatomie, de microchrurgie et chirurgie expérimentale, faculté de médecine et de pharmacie de Rabat.

\section{Manuscript Info} ........................

Manuscript History

Received: 08 June 2019

Final Accepted: 10 July 2019

Published: August 2019

Key words:-

Squamous cell carcinoma, heel, removal, groin and popliteal dissection.

\section{Abstract}

Introduction: There are malignant tumors of the skin with metastatic potential. We describe the case of a patient with a tumor of the heel who benefited of a surgical treatment in our unit.

Observation: we describe the case of a patient aged 48 years old who has a heel tumor measuring $10 \mathrm{~cm}$. The preoperative evaluation showed multiple lymph nodes in the groin and the popliteal fossa. We have performed a complete resection of the tumor immediately followed by groin and popliteal lymph node dissection. The reconstruction of the defect left by the removal of the heel tumor was done 15 days later with a skin graft.

Discussion: These carcinomas are often due to sun exposure. They are lymphophilic tumors. The removal of the tumor must be accompanied by lymph node dissection if the staging reveals lymphadenopathy. The groin dissection requires identification of the cleaning limits (femoral triangle), a vascular control, and removal of the cell-node lamina located around the saphenous vein. Two early complications are to remember: seroma and infection.

Conclusion: The treatment of our patient was made by the complete removal of the tumor with a groin and popliteal dissection. Skin grafting has full coverage of the loss of substance of the heel.

Copy Right, IJAR, 2019,. All rights reserved.

\section{Introduction:-}

Les carcinomes épidermoïdes cutanés sont des tumeurs malignes de la peau à potentiel métastatique. Ce sont des tumeurs qui envoient des métastases par voie lymphatique. Nous décrivons le cas d'un patient qui présente une tumeur du talon de grande taille dont le bilan d'extension a montré des adénopathies inguinales et poplitées et qui a bénéficié d'un traitement chirurgical dans notre service.

\section{Observation:-}

Nous décrivons le cas du patient H. H., âgé de 48 ans, qui nous a été adressé par un confrère du service de traumatologie pour la prise en charge d'une énorme tumeur du talon droit. L'examen clinique nous montre un patient en bon état général, apyrétique avec des conjonctives normalement colorées. Il a pour antécédents une

Corresponding Author:-J. Hafidi.

Address:-Service de chirurgie plastique et des brûlés, hôpital Ibn-Sina, Rabat. 
notion de tabagisme à raison de 30 paquets-année et une appendicectomie quand il avait l'âge de 21 ans. L'examen du talon droit montre une énorme tumeur bourgeonnante en choux fleur de $10 \mathrm{X} 10 \mathrm{~cm}$, non infectée, évoluant depuis 1 an selon le patient. L'examen des aires ganglionnaires a révélé de multiples adénopathies de la région inguinale et une adénopathie de la région poplitée. Le reste de l'examen clinique est sans particularités. Le bilan d'extension local et locorégional objective la présence à l'échographie de multiples ganglions de la région inguinale et du creux poplité dont les plus volumineuses mesurent $11 \mathrm{~mm}$ au niveau inguinal et $9 \mathrm{~mm}$ au niveau poplité. Le bilan d'extension général appuyé par une TDM thoraco-abdomino-pelvienne est sans anomalies, hormis une fibrose pulmonaire. Le patient est opéré en décubitus dorsal, sous anesthésie générale. Après badigeonnage et champtage (figure 1), nous réalisons une exérèse monobloc de la tumeur avec une marge de sécurité carcinologique de $2 \mathrm{~cm}$ emportant un tissu sain en profondeur (figure 2,3). Après hémostase rigoureuse nous déposons une compresse bétadinée sur la zone d'exérèse. Nous abordons ensuite le curage inguinal. Le tracé a mis en évidence le bord interne du sartorius, très oblique en bas et en dedans, sur une ligne dirigée depuis l'épine iliaque antérosupérieure vers la partie supéro-antéro-interne du tibia. Ce tracé met aussi en évidence le bord externe du long adducteur. Le pouls fémoral est marqué d'une croix. Une ligne est tracée depuis cette croix vers l'intersection des 2 lignes précédentes. Une bande de peau est hachurée au feutre de part et d'autre de cette ligne avec une largeur de $7 \mathrm{~cm}$, la largeur va en décroissant de proximal en distal (figure 4). Après ouverture du fascia cribriformis, on préparera le curage par une exposition de ses limites : dissection du bord supérieur du triangle exposant l'arcade fémorale, dissection du bord externe par incision du fascia du muscle sartorius jusqu'à la pointe du Scarpa : on préservera le nerf sensitif fémorocutané (branche du nerf crural) en disséquant la lame cellulo-ganglionnaire jusqu'au pédicule fémoral en dedans. Dissection du bord interne en ouvrant l'aponévrose du long adducteur jusqu'à découvrir la veine fémorale à la pointe du triangle de Scarpa : une ligature section des pédicules nourriciers des muscles long adducteur et pectiné est nécessaire. Contrôle de la veine saphène interne à la pointe du triangle de Scarpa qui va servir de guide pour l'évidement ganglionnaire. Le curage proprement dit part des limites du curage pour converger vers la crosse de la veine saphène interne : exposition des vaisseaux fémoraux (la veine étant à la pointe du Scarpa quasiment masquée par l'artère) : on commence en bas à la pointe du Scarpa en exposant l'artère fémorale (avec à sa face antérieure le nerf saphène (branche du nerf crural)) ; on remonte progressivement vers la crosse de la saphène interne en notant que la disposition des 2 vaisseaux change avec positionnement de la veine en dedans de l'artère au niveau de la crosse de la saphène ; on contrôle la crosse de la saphène interne au ras de la veine fémorale : la lame celluloganglionnaire n'est plus fixée que par les ganglions profonds situés au contact de la face interne de la veine fémorale remontant vers l'arcade fémorale . Evidement profond, le prélèvement intéresse les quelques ganglions reposant sur le muscle pectiné et situés à la partie interne de la veine fémorale, en particulier le ganglion de Cloquet le plus haut situé franchement sous l'arcade crurale. Après vérification de l'hémostase, un drain aspiratif est mis en place (figure 5) et une suture sous-cutanée par points séparés de fils résorbables $2 / 0$ et cutanée par points séparés non ischémiants termine l'intervention. Le curage poplité se fait ensuite en abordant la région poplitée par une incision horizontale de $4 \mathrm{~cm}$. Après incision du fascia superficialis et de l'aponévrose poplitée, la veine poplitée est disséquée en prenant garde à ne pas léser le nerf tibial. La crosse de la petite saphène est individualisée et respectée. Nous procédons à l'exérèse du tissu cellulo-ganglionnaire emportant une grosse adénopathie mesurant environ $1 \mathrm{~cm}$. La peau est refermée en 2 plans. Les suites opératoires sont simples. Le patient est mis sous antalgiques (codéïne + paracétamol) pendant 5 jours et sous couverture antibiotique : amoxicilline protégée à la dose de $1 \mathrm{~g} * 2 / \mathrm{j}$ pendant 10 jours. Le pansement à la bétadine est changé tous les jours au niveau de la cicatrice du curage inguinal. A J+8 nous procédons à l'ablation des fils de la cicatrice du curage inguinal. A J+12, nous procédons à l'ablation du drain de redon. Au niveau du talon, des pansements gras sont changés quotidiennement pour permettre un bon bourgeonnement du tissu de granulation. A J+15 le patient est de nouveau opéré pour une couverture de la perte de substance du talon laissée par l'exérèse de la tumeur. Le patient est opéré en décubitus dorsal sous rachianesthésie. Après badigeonnage et champtage, le tissu de granulation est trituré superficiellement à l'aide d'une curette pour le faire saigner et pour l'aplanir. Nous infiltrons au sérum adrénaliné (1 cc d'adrénaline dans 500cc de sérum salé) la zone donneuse, située au niveau de la cuisse droite. Nous prélevons ensuite une greffe de peau mince à l'aide du dermatome électrique. La zone donneuse est recouverte d'un pansement bétadiné. Le greffon est trempé dans une cupule contenant du sérum et une ampoule de gentamycine $(160 \mathrm{mg})^{*}$. Il est ensuite fixé sur la zone receveuse du talon à l'aide d'agraffes et d'un bourdonnet. Une atelle platrée est ensuite mise en place pour immobiliser le membre. L'ablation du bourdonnet se fait à $\mathrm{J}+4$, le pansement est ensuite changé tous les 2 jours. L'ablation des agraffes se fait à $\mathrm{J}+15$ et l'atelle platrée est retirée.

\section{Résultat:-}

Aucune complication chirurgicale n'a été déplorée pour notre patient avec un recul de 1 mois et demi. La cicatrice du curage inguinal est restée propre, sans signe d'infection ni lâchage de suture (figure 6). La greffe de peau mince a 
permis la couverture de toute la perte de substance laissée par l'exérèse de la tumeur (figure 7), on note un petit bourgeon hypertrophique qui est traité par application de corticoïdes en pommade en cure courte. La zone donneuse cicatrise par épidermisation insulaire spontanée à partir du sous sol. Le compte rendu anatomopathologique de la pièce opératoire a mis en évidence un carcinome verruqueux du talon. Les limites chirurgicales sont indemnes. L'examen de la pièce du curage inguinal et poplité a mis en évidence 7 ganglions dont le plus grand mesure $1,7 \mathrm{~cm}$ de grand axe. Les 7 ganglions examinés montrent un parenchyme ganglionnaire indemne d'infiltration ganglionnaire $(0 \mathrm{~N}+/ 7 \mathrm{~N})$.

\section{Discussion:-}

Les carcinomes spinocellulaires cutanés, ou carcinomes épidermoïdes, sont des tumeurs malignes de la peau qui, en plus des basocellulaires, font partie du groupe des tumeurs cutanées épithéliales. Ils sont invasifs localement et ont le potentiel de faire des métastases. Ils sont par ordre de fréquence la deuxième forme de cancer cutané dans le monde entier. Leur incidence varie en fonction de la région géographique et de l'origine ethnique, et la plupart des études en constatent une augmentation mondiale $[1,2]$. L'exposition solaire cumulative est le principal facteur causal des carcinomes épidermoïdes. Le rôle des HPV oncogènes est suspecté chez le patient immunocompétent, où l'infection par les HPV prédispose aux carcinomes des muqueuses [3]. Ils ont un type d'expansion infiltratif et peuvent envahir les fascias, le périoste, le périchondre et les gaines périneurales. Ils peuvent faire des métastases locales, régionales et à distance, $80 \%$ par le système lymphatique [2]. Ils surviennent souvent sur une lésion précancéreuse (précurseur). $\mathrm{Au}$ niveau cutané Les kératoses photo-induites (kératoses actiniques ou solaires ou « séniles ») sont les lésions précancéreuses les plus fréquentes. Elles peuvent régresser spontanément ou évoluer en un authentique carcinome spinocellulaire. Il existe un continuum entre la kératose actinique, le carcinome in situ et le carcinome invasif. Elles siègent sur les zones photoexposées (visage, dos des mains) : aspect de lésions croûteuses souvent multiples, plus ou moins érythémateuses, qui saignent facilement après grattage. Au niveau muqueux, les leucoplasies qui résultent d'un phénomène de kératinisation de la muqueuse buccale, en particulier labiale, le plus souvent due au tabac ou aux UV (chéilite actinique), sont des lésions blanchâtres bien limitées, asymptomatiques, adhérentes et ne saignant pas au contact. Certains états inflammatoires chroniques muqueux rares peuvent se transformer avec une relative fréquence : le lichen scléreux génital et le lichen érosif buccal. Le carcinome intraépithélial ou carcinome in situ ou maladie de Bowen est la première étape non invasive du carcinome épidermoïde (pas de franchissement de la membrane basale). Au niveau cutané cela se traduit par une lésion rare, le plus souvent unique, souvent sur les zones photoexposées, prenant l'aspect d'une lésion érythémateuse plus ou moins pigmentée et croûteuse toujours bien limitée [4]. Sur le plan clinique, le carcinome épidermoïde est une tumeur dont le siège peut être cutané ou muqueux qui prend la forme d'une lésion croûteuse, jaunâtre, indurée avec ulcération centrale ou d'une lésion végétante ou bourgeonnante ou parfois d'une association des deux. Quel qu'en soit l'aspect clinique, cette lésion chronique, ulcérée ou nodulaire doit faire pratiquer une biopsie qui va permettre de confirmer le diagnostic par l'analyse histologique. Il existe plusieurs types histologiques : verruqueux, acantholytique/ adénoïde, à cellules fusiformes, baso-squameux, pseudovasculaire, muco-épidermoïde/ adénosquameux, desmoplastique [5]. Leur traitement est toujours l'exérèse chirurgicale avec, dans toute la mesure du possible, une marge de sécurité clinique de $1 \mathrm{~cm}$ dans les formes débutantes, emportant en profondeur une barrière anatomique saine [6]. Pour les tumeurs avec risque élevé de récidive et dans les formes évoluées, surtout muqueuses, la marge de sécurité peut atteindre 3 à $5 \mathrm{~cm}$. La chirurgie contrôlée par micrographie selon Mohs prend toujours plus d'importance dans le traitement chirurgical. En alternative à l'excision chirurgicale, et après confirmation histologique, il est possible d'envisager la radiothérapie. Elle n'entre en ligne de compte comme premier traitement que pour les patients chez lesquels un traitement chirurgical est impossible en raison de contre-indications relatives, que ce soit sur leur désir ou en cas de localisations chirurgicales compliquées comme au niveau de l'angle palpébral, du nez ou de la lèvre. La radiothérapie peut également être une option pour des tumeurs à mauvais pronostic (récidivants ou à un stade avancé). Elle peut également être indiquée pour les formes dépassées, après chirurgie de propreté [2, 3].

Le triangle de Scarpa ou trigone fémoral est situé à la racine de la partie antéro-interne de la cuisse. Il présente la forme d'une pyramide triangulaire à base supérieure constitué par l'espace inter-ilio-pariétal et à sommet inférieur correspondant à l'orifice supérieur du canal fémoral. La limite supérieure correspond à l'arcade crurale. Le triangle de Scarpa est limité en dehors par le bord interne du muscle sartorius. Ce dernier naît au niveau l'épine iliaque antéro-supérieure, croise le muscle psoas, la partie inférieure du pectiné et la face antérieure du long adducteur, il se dirige en bas et en dedans vers la face interne de la cuisse et se termine sur la partie antéro-supéro-interne du tibia. La limite interne du triangle de Scarpa est le bord externe du muscle long adducteur. Il s'insère en haut sur l'angle du pubis et se termine en bas et latéralement sur la partie supérieure de la ligne âpre du fémur. Le fond ou plancher du triangle de Scarpa est tapissé par 2 muscles : le psoas-iliaque en dehors et le pectiné en dedans. Le muscle psoasiliaque est représenté par sa partie inférieure et son tendon terminal. Il passe sous l'arcade crurale dans sa moitié 
externe en dehors de la bandelette ilio-pectinée. Il est enveloppé dans une gaine : le fascia iliaca qui contient aussi le nerf crural. Il se termine sur la partie post du petit trochanter. Le muscle pectiné s'étend entre la crête pectinéale, la lèvre antérieure de la gouttière sous pubienne et la branche moyenne de trifurcation de la ligne âpre. La paroi antérieure ou plafond du triangle de Scarpa comprend la peau, elle est fine, glabre et adhère par sa face profonde à l'arcade crurale. L'aponévrose fémorale superficielle ou fascia cribriformis est tendue entre les 3 bords du triangle dont elle forme le couvercle [7]. Le sommet du triangle de Scarpa est inférieur et correspond à l'orifice supérieur du canal crural (ou fémoral), il est situé au point de croisement du sartorius et du long adducteur. Le contenu du triangle de Scarpa comprend un plan superficiel et un plan profond. Le plan superficiel inclus des vaisseaux superficiels : la veine saphène interne, elle longe le bord médial, décrit une crosse traversant le fascia cribriformis pour gagner la veine fémorale commune (plan profond). La crosse reçoit 4 veines collatérales satellites à leurs artères homonymes (branches de l'artère fémorale commune) : la sous cutané abdominale en dedans, la circonflexe iliaque superficielle en dehors et les honteuses externes supérieur et inférieur. Les nerfs superficiels comprennent les branches nerveuses perforantes du nerf crural (ou fémoral), le nerf fémoro-cutané en dehors, le rameau crural du nerf génito-crural à la partie moyenne et le rameau superficiel de l'accessoire du nerf saphène interne en dedans. Les lymphatiques superficiels sont formés par une série de troncs lymphatiques qui cheminent en dedans de la veine saphène interne et se jettent dans les ganglions inguinaux superficiels, répartis autour de la crosse en 4 groupes. Le plan profond du triangle de Scarpa contient des vaisseaux profonds : l'artère fémorale commune en dehors, elle fait suite à l'artère iliaque externe au niveau de l'arcade crurale, traverse verticalement tout le trigone et se divise en fémorale profonde et superficielle. Elle donne 4 branches collatérales avant sa division. On distingue également l'artère fémorale profonde, branche postérieure de la fémorale commune. Elle est oblique en dehors et en arrière. Volumineuse, elle irrigue les muscles et les téguments de la presque totalité de la cuisse. Elle passe dans la loge des adducteurs où elle donne des collatérales pour le trigone : l'artère du muscle quadriceps, destinée aux 4 chefs de ce muscle et au muscle sartorius, l'artère circonflexe antérieure qui s'anastomose avec l'artère circonflexe postérieure, l'artère circonflexe postérieure, elle s'anastomose avec les artères circonflexe antérieure ,les artères ischiatique et première perforante ; et aussi les artères perforantes qui traversent les insertions des muscles adducteurs et pénètrent dans la région postérieure de la cuisse. L'artère fémorale superficielle quant à elle continue son trajet verticalement. Elle passe dans le canal crural et donne une seule branche, l'artère grande anastomotique au niveau de l'anneau du grand adducteur puis elle devient l'artère poplitée. La veine fémorale fait suite à la veine poplitée et s'étend jusqu'à l'anneau crural pour devenir veine iliaque externe. Elle croise de dehors en dedans en passant en arrière l'artère et reçoit la crosse de la veine saphène interne et la veine fémorale profonde. Les Nerfs profonds comprennent le rameau crural du nerf génito-crural et le nerf fémoro-cutané et surtout le nerf crural ou fémoral. Il donne une branche collatérale : le nerf de l'artère fémorale et 4 branches terminales disposées sur 2 plans, l'un superficiel, formé par les nerfs musculo-cutanés interne et externe et l'autre profond, formé par le nerf du muscle quadriceps qui se divise en 4 branches motrices et le nerf saphène interne. Les lymphatiques profonds cheminent en dedans de la veine fémorale et se jettent dans les ganglions inguinaux profonds au niveau de l'infundibulum crural, entre la crosse de la saphène interne et l'anneau crural [8].

Le curage inguino-fémoral est indiqué en cas de carcinome spinocellulaire accompagné d'adénopathies métastatiques. Ses impératifs sont l'identification des limites du curage (triangle de Scarpa), un contrôle vasculaire, et une exérèse de la lame cellulo-ganglionnaire centrée sur la crosse de la saphène interne de manière concentrique [9]. Deux complications précoces dominent lors des suites post-opératoires: les lymphocèles et l'infection. Les lymphocèles postopératoires sont fréquents et imposent des ponctions itératives si le drain aspiratif a été retiré trop hâtivement ; le lymphocèle est susceptible de s'infecter et est source de souffrance cutanée elle-même responsable de nécrose (avec une cicatrisation dirigée longue et pénible pour la patiente) [10]. Pour diminuer le risque de lymphocèle, certains auteurs préconisent de recouvrir les vaisseaux fémoraux en transposant le muscle sartorius sectionné à son origine au plus près de l'épine iliaque antéro-supérieure et fixé en dedans sur l'arcade crurale. Cependant un essai randomisé récent portant sur 28 curages avec transposition et 33 curages sans n'a pas retrouvé de bénéfice après ajustement à ce geste, voire même une augmentation des lymphocèles postopératoires [11]. La deuxième complication est l'infection postopératoire. Après prélèvements bactériologiques pour une antibiothérapie adaptée, le traitement consiste en une mise à plat et drainage dont les conséquences sur la plaie peuvent être amoindries si l'on a pris la précaution d'une fermeture par points séparés. Les autres complications précoces sont la nécrose cutanée ischémique, les hématomes postopératoires qui imposent une reprise chirurgicale pour assurer l'hémostase et les phlébites des membres inférieurs. Les complications tardives sont les hernies crurales qui peuvent être prévenues par l'abaissement de l'arcade crurale sur le ligament de Cooper, les lymphœdèmes des membres inférieurs : ils sont d'autant plus fréquents que le curage était important, que le nombre de ganglions métastatiques était élevé, qu'une radiothérapie inguinale et pelvienne a succédé à la chirurgie, que les suites postopératoires ont été 
agitées ; comme pour le membre supérieur, une mobilisation précoce, une contention élastique intéressant la cuisse et la surélévation modérée du membre sont conseillés comme mesures préventives ; lorsque le lymphœdème est installé, il est nécessaire de le traiter à vie par des séances de drainage lymphatique manuel ou utilisant des appareils d'endermologie régulières associées aux mesures préventives sus-citées [12].

\section{Conclusion:-}

Le traitement du carcinome épidermoïde cutané chez notre patient s'est fait par l'exérèse complète de la tumeur accompagnée d'un curage ganglionnaire inguinal et poplité. La reconstruction de la perte de substance laissée par l'exérèse de la tumeur s'est faite à distance par une greffe de peau mince et a permis une couverture complète de la perte de substance du talon.

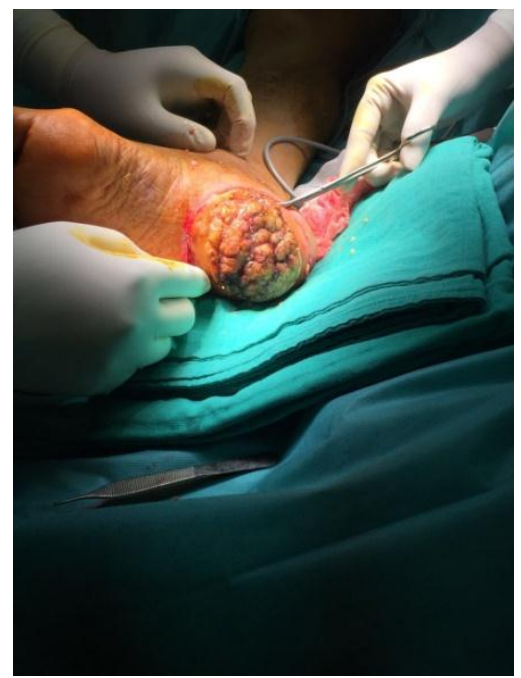

Fig 1:-Tracé de l'incision, une marge de $2 \mathrm{~cm}$ est respectée autour de la tumeur.

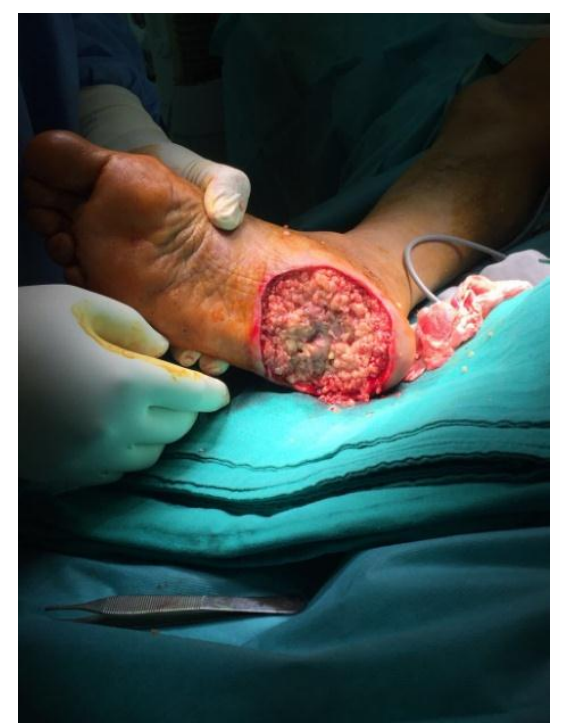

Fig 2:-Aspect du talon après exérèse de la tumeur. 


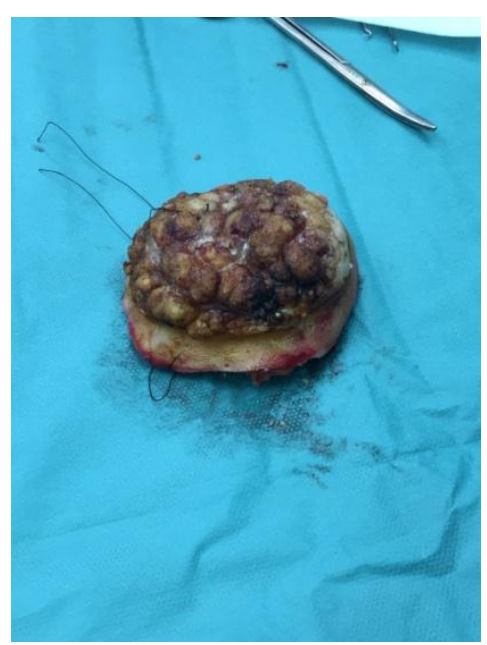

Fig 3:-Aspect de la pièce opératoire après exérèse. Elle est adressée au laboratoire d'anatomopathologie. La pièce est orientée par 2 fils à midi et un fil à 9 h.

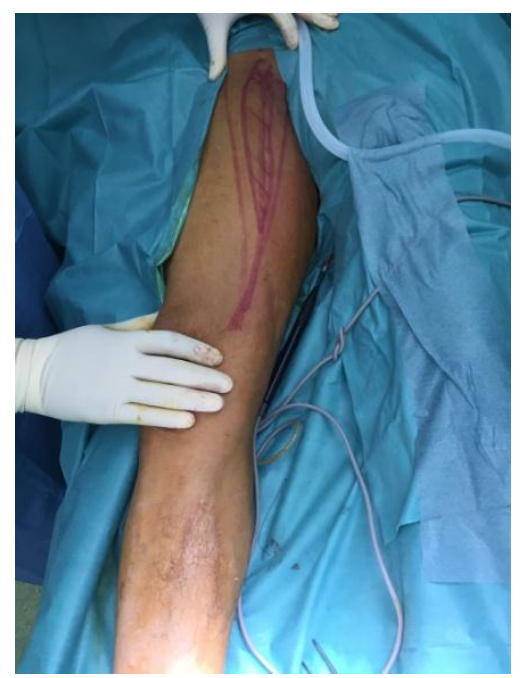

Fig 4:-Tracé des incisions pour le curage inguinal.

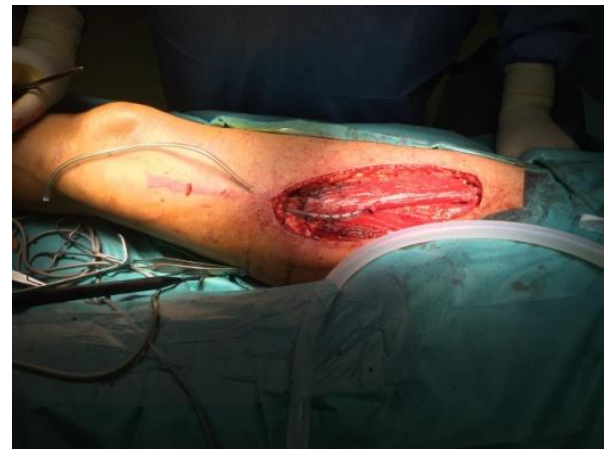

Fig 5:-Triangle de Scarpa après curage et hémostase rigoureuse. Un drain de redon est mis en place. 


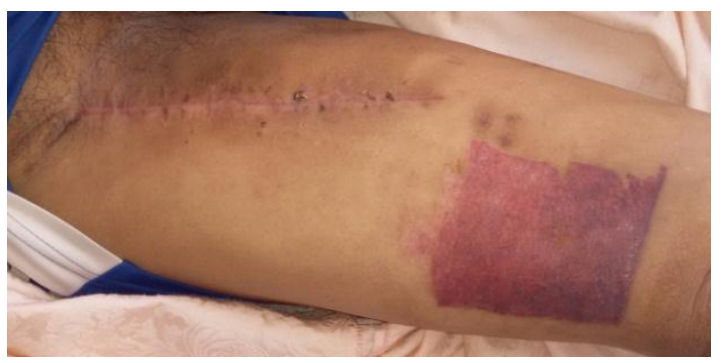

Fig 6:-Aspect de la cicatrice du curage inguinal et de la zone donneuse à $\mathrm{J}+1$ mois et demi

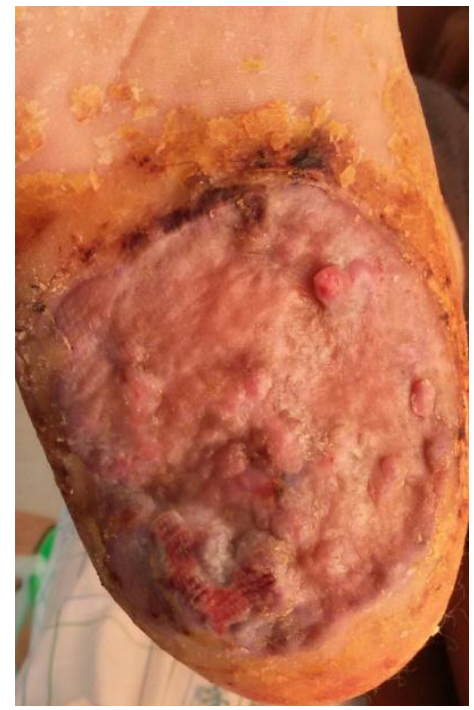

Fig 7:-Reconstruction du talon par une greffe de peau mince. Résultat à $\mathrm{J}+1$ mois et demi

\section{Références:-}

1. Ridky TW. Nonmelanoma skin cancer. J Am Acad Dermatol. 2007; 57(3):484-501.

2. Bonerandi JJ, Beauvillain C, Caquant L, Chassagne JF, Chaussade V, Clavere P, et al. Guidelines for the diagnosis and treatment of cutaneous squamous cell carcinoma and precursor lesions. J Eur Acad Dermatol Venereol. 2011;25 Suppl 5:1-51.

3. NCCN CpGiO. Basal cell and Squamous Cell Carcinoma. 2012

4. Edge SB, Compton CC. The American Joint Committee on Cancer: the 7th edition of the AJCC cancer staging manual and the future of TNM. Ann Surg Oncol. 2010;17(6):1471-4.

5. Motley R, Kersey P, Lawrence C. Multiprofessional guidelines for the management of the patient with primary cutaneous squamous cell carcinoma. Br J Dermatol. 2002;146(1):18-25.

6. M. Revol, JM Servant. Manuel de chirurgie plastique, reconstructrice et esthétique. 2ème edition revise et augmentée. 2009; 206-215.

7. Chevallier JM. Anatomie Flammarion Médecine Sciences. Paris, 1998.

8. Rouvière H. Anatomie humaine, descriptive et topographique. Masson. Paris, 1984 (12ème édition).

9. Querleu D. Chirurgie des cancers. Chirurgie de stadification. In: Masson, editor. Techniques chirurgicales en gynécologie. 2e édition. Paris 1998;p125-7.

10. Spratt J. Groin dissection. J Surg Oncol 2000;73:243-62.

11. Judson PL, Jonson AL, Paley PJ, Bliss RL, Murray KP, Downs LS Jr, Boente MP, Argenta PA, Carson LF. A prospective, randomized study analyzing sartorius transposition following inguinal-femoral lymphadenectomy. Gynecol Oncol 2004;95:226-30.

12. Société Française d'Anesthésie Réanimation. Recommandations pour la pratique clinique. Prévention de la maladie thromboembolique veineuse périopératoire et obstétricale. 2005. 